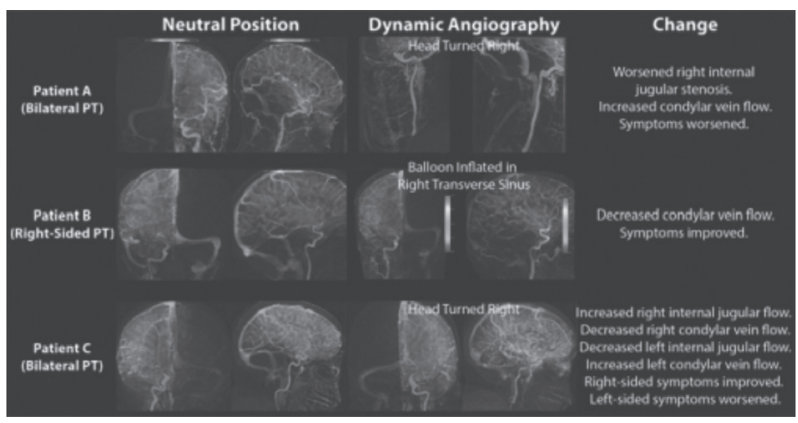

Abstract P024 Figure 1

prominent condylar veins and means to localize symptoms to these structures using dynamic angiography with head positioning and venous balloon test occlusion.

Materials and methods Retrospective analysis was performed under IRB approved protocol for 43 consecutive PT patients referred for diagnostic angiography at a major academic medical center between January 2013 and December 2015. Rates of different etiologies of PT among patients in this group were noted. In addition to standard diagnostic cervicocerebral angiography, dynamic angiography with head turning was conducted. The effects of provocative maneuvers were measured using time-resolved flow analysis known (iFlow, Siemens Healthcare, Erlangen Germany).

Results 5 (11.6\%) patients were found to have prominent condylar veins. 16 (37.2\%) had a dAVF, 4 (9.3\%) had a venous diverticulum, and 9 (20.9\%) had no angiographic abnormality detected. The findings of the five patients with prominent condylar veins are summarized in the table. Representative images are provided in the figure.

Conclusion Prominent flow in condylar veins should be considered among the numerous causes of PT. This may be a frequent cause of these symptoms in patients who previously would have had no identifiable cause Further investigation is warranted with respect to this cause of PT and PT in general. Disclosures M. Alexander: None. K. Meisel: None. V. Halbach: None. R. Darflinger: None. A. Nicholson: None. F. Settecase: None. D. Cooke: None. R. Higashida: None. C. Dowd: None. S. Hetts: None. M. Amans: None.

\section{P-025 CALCIUM CHANNEL BLOCKER INFUSION IMPROVES VESSEL MEASUREMENT ACCURACY AND ANEURYSM OCCLUSION FOLLOWING FLOW DIVERSION}

M Alexander, R Darflinger, F Settecase, A Nicholson, D Cooke, M Amans, S Hetts, C Dowd, R Higashida, V Halbach. Radiology and Biomedical Imaging, UCSF, San Francisco, $C A$

\subsection{6/neurintsurg-2016-012589.67}

Introduction/purpose To best achieve complete occlusion of aneurysms treated with flow diverters (FDs), devices must be accurately sized to precisely match the parent vessel diameter. Appropriately sized FDs will have maximal coverage over the aneurysm neck and will be more likely to induce thrombosis. Catheterization for diagnostic angiography can cause vasospasm that may affect vessel measurements. This study evaluates the effects of intra-arterial infusion of a calcium channel blocker (CCB) on angiographic measurements in patients treated with FDs, investigating the impact on the final diameter of the expanded stent following deployment and subsequent occlusion.

Materials and methods Retrospective analysis was performed of prospectively maintained procedure records to identify patients with aneurysms treated with FDs. In all patients, pre-treatment measurements were recorded for vessel diameter of the distal and proximal landing zones, as well as maximum and minimum diameters between these segments. Post-treatment measurements of the stent itself following deployment were recorded at the distal and proximal ends in addition to maximum and minimum diameters. When $\mathrm{CCB}$ was infused prior to treatment, post-infusion pre-treatment measurements were recorded. All measurements were performed after calibrating to a catheter included on the image. Rates of occlusion and time to confirmed occlusion were noted for all patients. Independent-sample 2 tailed T-tests were performed to assess for differences in pre- and post-treatment measurements, rates of occlusion, and time to confirmed occlusion between groups with and without CCB infusion.

Results 21 FDs were deployed to treat 19 aneurysms in 18 patients. CCB infusion was performed prior to deployment of 9 devices. The change in measurements noted after CCB infusion are summarized in Table 1 . No significant difference was noted between groups for pre- and post-treatment measurement changes. Subsequent aneurysm occlusion was more likely

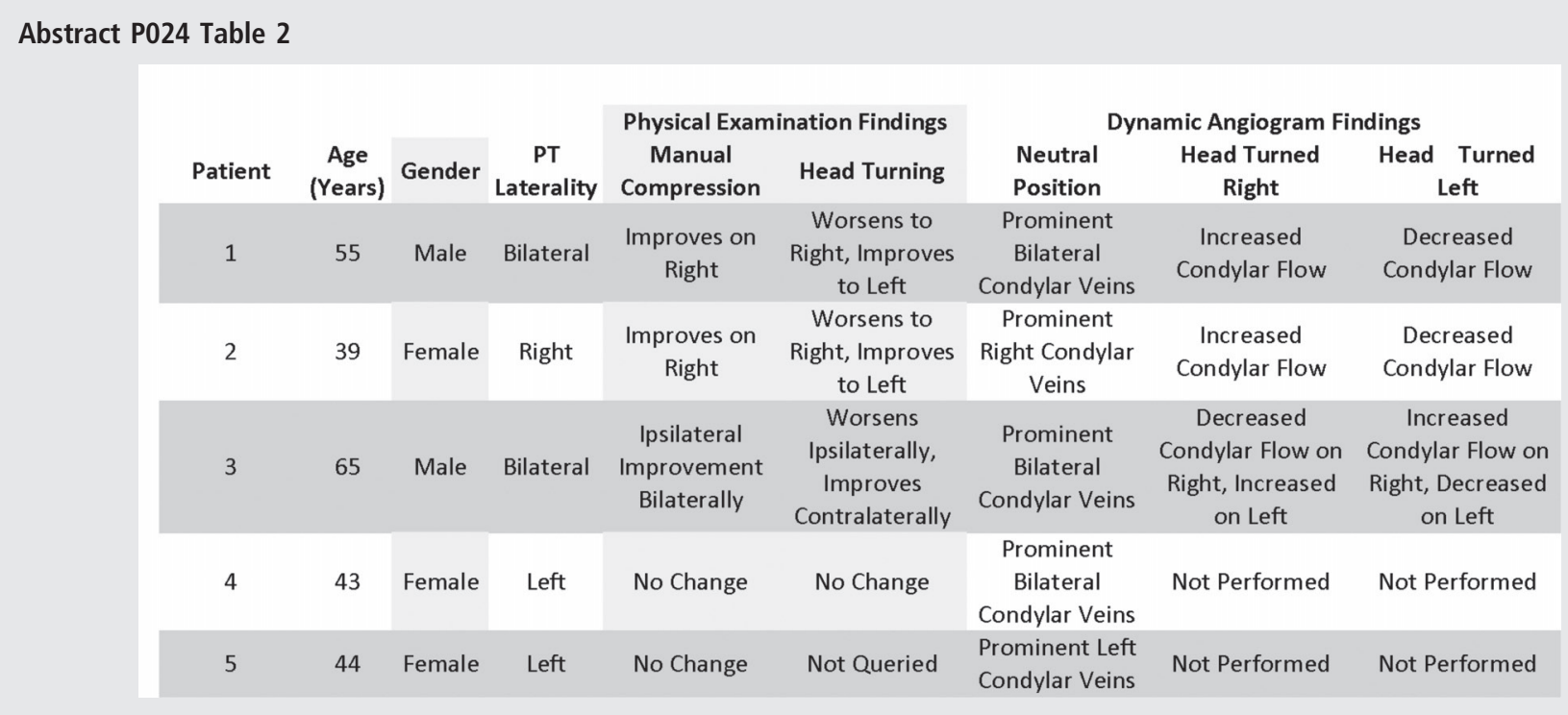


to occur in the CCB infusion group $(100 \%$ vs. $44.4 \%$, $\mathrm{p}<0.001)$. No significant difference was noted between groups for time to occlusion (313.7 vs. 392.8 days, $\mathrm{p}=0.807)$ or overall angiographic follow up time (209.1 vs. 302.5 days, $\mathrm{p}=0.326$ ).

Conclusion Optimization of device sizing is important to increase FD density over the aneurysm neck and promote thrombosis. To improve accuracy of measurements of parent vessels prior to device selection, CCB infusion can reduce the effects of mild vasospasm. In this study, subsequent aneurysm occlusion was more likely to occur following FD treatment when device size selection was based on measurements performed following CCB infusion.

\begin{tabular}{lc} 
Abstract P-025 Table 1 & Diameter change after CCB infusion \\
\hline Measurement & Change \\
\hline Distal Landing Zone & $30.2 \%$ \\
Proximal Landing Zone & $44.3 \%$ \\
Maximum Diameter & $60.7 \%$ \\
Minimum Diameter & $8.4 \%$ \\
\hline
\end{tabular}

Disclosures M. Alexander: None. R. Darflinger: None. F. Settecase: None. A. Nicholson: None. D. Cooke: None. M. Amans: None. S. Hetts: None. C. Dowd: None. R. Higashida: None. V. Halbach: None.

\section{P-026 CERVICAL CAROTID "PSEUDO-OCCLUSION:" INTRACRANIAL OCCLUSIONS MASQUERADING AS EXTRACRANIAL OCCLUSIONS}

${ }^{1} \mathrm{~J}$ Grossberg, ${ }^{2} \mathrm{~F}$ Cardoso, ${ }^{2} \mathrm{~L}$ Rebello, MD, ${ }^{2} \mathrm{D}$ Haussen, ${ }^{2} \mathrm{M}$ Frankel, ${ }^{2} \mathrm{R}$ Nogueira. ${ }^{1}$ Department of Neurosurgery, Emory University School of Medicine, Atlanta, GA; ${ }^{2}$ Emory, Atlanta, GA

10.1136/neurintsurg-2016-012589.68

Introduction The entity of internal carotid artery (ICA) "pseudo-occlusion," where an occlusion of the intracranial ICA appears as an occlusion of the extracranial cervical ICA on computed-tomography angiography (CTA) or digital subtraction angiography (DSA) is more common than previously thought. We aim to accurately describe and analyze this entity for the benefit of the treating interventionalist.

Methods This was a retrospective review of a prospectively collected thrombectomy database between February 2011-January 2016. Over 900 patients were treated within the study period, and we subselected the 46 patients who had an occlusion of the intracranial ICA without evidence of a tandem occlusion in the cervical ICA. These patients' angiograms were analyzed for the presence of cervical ICA "pseudo-occlusion." Details of their demographics, medical history, thrombectomy procedure, and outcome were then analyzed.

Results The mean age for the total population was 62.3 (Range: 31-91). There was an exact split between sexes in the total population. Cervical ICA "pseudo-occlusion" was found in 21 of the 46 patients (46\%) on CTA. 17 (81\%) of these patients had "pseudo-occlusion" of the proximal cervical ICA and 4 had "pseudo-occlusion" of the mid-cervical ICA. $15(71 \%)$ of these patients continued to have "pseudo-occlusion" on DSA during their thrombectomy procedure.
When comparing the two groups, $48 \%$ of the "pseudoocclusion" group and 52\% of the non-"pseudo-occlusion" group were male. The mean age of the "pseudo-occlusion" cohort was 64.8 versus 60.1 in the non-"pseudo-occlusion" cohort. $38 \%$ of the "pseudo-occlusion" patients and $40 \%$ of the non-"pseudo-occlusion" patients received IV TPA.

The rates of mTICI $2 \mathrm{~b} / 3$ reperfusion were $81 \%$ in the "pseudo-occlusion cohort" versus $100 \%$ in the non-"pseudoocclusion" cohort. ( $p<0.05)$. The mean procedure length was 89.45 minutes in the "pseudo-occlusion" group

versus 62.16 minutes in the non-"pseudo-occlusion" group $(\mathrm{p}=0.07)$. The rate of ECASS Parenchymal Hematoma Type 2 was $4.8 \%$ in the "pseudo-occlusion" group versus $4 \%$ in the non-"pseudo-occlusion" group. 90 day follow-up mRS was available in 20 "pseudo-occlusion" patients and 19 non"pseudo-occlusion" patients. The rate of 90 day mRS 0-2 was $35 \%$ in the "pseudo-occlusion" group and $63 \%$ in the non"pseudo-occlusion" group. The 90 day mortality rate was $25 \%$ in the "pseudo-occlusion" group and $21 \%$ in the non"pseudo-occlusion" group.

Conclusions Carotid "pseudo-occlusion" is a common entity in the thrombectomy population, and its presence is associated with increased procedure time and decreased rates of reperfusion.

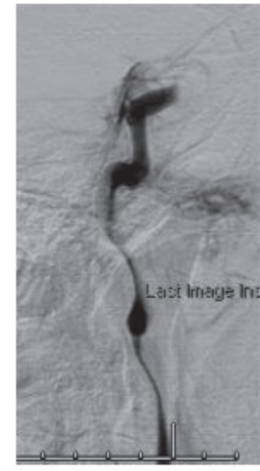

DSA

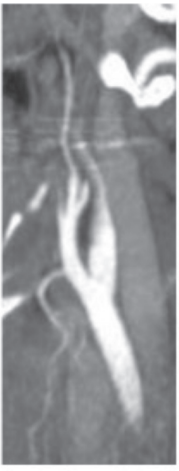

CTA
Abstract P-026 Figure 1

Disclosures J. Grossberg: None. F. Cardoso: None. L. Rebello: None. D. Haussen: None. M. Frankel: None. R. Nogueira: None.

\section{P-027 EXPERIMENTAL TESTING OF DIFFERENT TYPES OF WOVEN ENDOBRIDGE DEVICES IN ELASTASE-INDUCED ANEURYSMS IN RABBITS}

Y Ding, D Dai, D Schroeder, R Kadirvel, D Kallmes. Radiology, Mayo Clinic, Rochester, MN

\subsection{6/neurintsurg-2016-012589.69}

Purpose The Woven Endobridge (WEB) device (Sequent Medical, Inc., Aliso Viejo, CA) is an intrasaccular flow-disruption device. The purpose of this study was to evaluate the acute and chronic performance of new generation WEB devices using rabbit aneurysm model.

Materials and methods Six Enhanced Visualization (EV) WEBDL (Dual Layer with barrel configuration); six EV WEB-SL (Single Layer with barrel configuration); and six EV WEB-SLS 